\title{
Transverse structure of the wave function of field emission electron beam determined by intrinsic transverse energy
}

\author{
Soichiro Tsujino ${ }^{\text {a) }}$ \\ Laboratory for Micro- and Nanotechnology, Photon Science Division, Paul Scherrer Institut, \\ 5232 Villigen-PSI, Switzerland
}

(Received 13 April 2018; accepted 6 July 2018; published online 24 July 2018)

\begin{abstract}
The average transverse energy of field emission electrons at the cathode surface is one of the key factors that determines the virtual source size, hence the transverse spatial coherence of field emitters. In the past, the subject has been intensively studied by classical electron optics analysis but its wave optical studies are rare. In this work, we therefore aim to elucidate the influence of the transverse momentum in solid on the transverse structure of the wave function of field emission electrons. From the calculation extending the standard field emission theory within the WKB approximation for model planar free-electron metal, we obtained a Gaussian-beam-type wave function that exhibits a minimum transverse width at the cathode surface as determined by the average transverse energy and propagates the first few nanometers with a limited transverse spread. At far field, the wave function spreads as the electron propagates away from the cathode surface. Comparison with classical results indicated that, in the present planar field emitter model, the neglect of the three-dimensional potential around the tip apexes of actual field emitters underestimates the transverse spread up to a factor of 2 . However, when the cathode size is finite and the electrons in the solid are phase-coherent within the source area, the transverse spread is much smaller than that of the point-source wave function. Our result indicates that the intrinsic transverse emittance of a finite size fully coherent field emitter is much smaller than the value predicted by classical analysis. Published by AIP Publishing. https://doi.org/10.1063/1.5035284
\end{abstract}

\section{INTRODUCTION}

Field emitters have been successfully utilized in the past for high resolution electron microscopy and crystallography, ${ }^{1-3}$ coherent electron imaging, ${ }^{4-8}$ as well as in wave front control experiments ${ }^{9-11}$ by taking advantage of their high beam brightness and the large transverse coherence length. Because of the requirement of the high surface electric field in the order of a few $\mathrm{GV} / \mathrm{m}$, the apex radius of a field emitter is often reduced to nanometer range. This is for the purpose of decreasing the required voltage to practical levels by utilizing the field enhancement at sharp protrusions. The resulting small source size in turn has an effect to increase the transverse spatial coherence length as the van Cittert-Zernike theorem predicts. ${ }^{12}$ At the same time, the fact that the physical source size of field emitters is normally much larger than the virtual source size is well known. ${ }^{13-16}$ Experimentally, the virtual source size is evaluated from the transverse coherence length as determined, e.g., from the interference experiments using the electron biprism ${ }^{17-19}$ and applying the van Cittert-Zernike theorem assuming that electrons emitted from different parts of the field emitter surfaces are uncorrelated.

As recognized in the literature of the classical electron optics of field emitters, ${ }^{13,14,20-22}$ the small virtual source size is partly a consequence of the curved emitter apex: when the trajectories of field emission electrons emitted from an emitter tip apex surface are extrapolated into the solid, they

${ }^{\text {a)} E l e c t r o n i c ~ m a i l: ~ s o i c h i r o . t s u j i n o @ p s i . c h ~}$ converge around the center of the tip apex radius of curvature, where an effective virtual source (the so-called "crossover") is formed. However, even in the case of a perfectly spherical emitter tip apex, the virtual source size is finite since the finite transverse energy spread of the field emission electron ${ }^{13,14,20-22}$ causes the deflection of electron trajectories from the radial surface-normal directions. The average transverse energy is also a determinant factor of the resolution of the field emission microscope $e^{21,23}$ together with the magnification and the quantum mechanical spatial uncertainty of the electrons that will be increasingly important for smaller field emitter tips.

These investigations have established the electron optical properties of field emitters that are important for several practical applications. However, for quantitative modeling and analysis of experiments such as the electron diffraction of large unit cell samples (e.g., protein crystals) and coherent imaging of specimens with finite thicknesses, it is crucial to know the transverse structure of the wave function since the transverse structure of the field emission electron wave function would determine the transverse spatial coherence length of the field emission beam. The transverse structure of the wave function also has a large impact on the recently studied ultrafast electron dynamics at the field emitter apex, where the large amplitude oscillating electric field of a few cycle laser pulses induces the field emission and forced oscillation and re-scattering at the tip apex, resulting in a rich variety of nanoscale strong field physics. ${ }^{24-27}$ These effects might be significantly diminished unless the transverse spread of the wave function during the propagation near the tip apex was 
restricted. Despite the prior success of the wave optical analysis of such experiments by using the theory of the partially coherent optical beams, ${ }^{28-30}$ experiments are often in a diode configuration, in which electrons emitted from the cathodes are accelerated toward the samples or anodes, hence, such optical theory neglecting the acceleration is of limited validity. Further we note that, considering the uncertainty relationship between the position and the momentum in the transverse direction, we anticipate for a point source field emitter that the electron wave function has a finite transverse spread $\sigma_{0}$ in the order of $\sim \hbar / p_{t}$, where $\hbar=h / 2 \pi$ ( $h$ is the Planck's constant) and $p_{t}$ is the average transverse momentum as given by the average transverse energy $\left\langle E_{t}\right\rangle=p_{t}^{2} /(2 m)$. To calculate $\sigma_{0}$ as well as the evolution of the transverse spread of the wave function under acceleration and the wave front curvature of the electron wave, one should study the three-dimensional solution of the Schrödinger equation of field emission electrons in the acceleration potential from the surface of the cathode. These are beyond the classical electron optics.

We note that recent advancements of the acceleratorbased high brightness $\mathrm{X}$-ray sources, such as the $\mathrm{X}$-ray freeelectron lasers, ${ }^{31-33}$ demand technological developments of extremely high beam brightness cathodes, as characterized not only by the high electron flux density but also by the small intrinsic transverse emittance of the cathode. Such high brightness cathodes are demanded also for ultrafast electron diffraction experiments. ${ }^{34-36}$ Motivated by these backgrounds, the laser-induced field emission from needleshaped etched wire field emitters ${ }^{37-39}$ and the surface-plasmon-enhanced laser-induced field emission from doublegate metal nanotip array cathodes ${ }^{40-43}$ have been intensely studied recently. Since the intrinsic transverse beam emittance is given by the cathode size and the intrinsic transverse beam spread is determined by the average transverse energy of the produced electron beam, ${ }^{44}$ there is a renewed interest in the intrinsic transverse emittance and the transverse average energy of field emission beam in particular, for accelerator applications. ${ }^{31,45}$ When the field emitter is applied for electron diffraction experiments, not only the high beam brightness but also the long transverse coherence is important. These two quantities are closely related as exemplified in literature but their quantum mechanical implication is yet to be studied.

Accordingly, the purpose of this work is to apply the constraints of electronic states in the solid on the solution of the Schrödinger equation of field emission electron beam. The result shows that the average transverse energy defines the minimal transverse width of field emission electron beam that is independent from the effect of the quantum confinement. Recently, Edgcombe considered the transverse structure of the wave function of field emission electrons. ${ }^{46,47}$ Their solution suggests that the transverse spread of the wave function of the field emission electron during the propagation is strongly restricted. However, they did not take into account the constraints on the possible solutions by the limited available electronic states in the solid upon tunneling the surface barrier. We found that this constriction should be taken into consideration as shown below.
This article is organized as follows. In Sec. II, we first summarize the result of the standard field emission theory for the calculation of the current density and the average energy spreads for completeness. Then in Sec. III, we discuss the calculation of the wave function of the field emission electron by extending the standard field emission theory. In Sec. IV, we discuss the implication of the thus obtained wave function, and finally summarize in Sec. V.

\section{SUMMARY OF THE RESULTS OF THE STANDARD FIELD EMISSION THEORY}

We consider a planar metal residing in $z<0$ and examine the field emission of electrons from the metal surface at $z=0$. We assume that electrons in the metal are free-electron like with the electron effective mass equal to the electron rest mass $m_{0}$, and their density is characterized by the Fermi energy $E_{F}$ in the order of $5-10 \mathrm{eV}$ (with the corresponding Fermi wavelength $\lambda_{F}$ equal to 5.5 and $3.9 \AA$ at $E_{F}=5$ and $10 \mathrm{eV}$, respectively). We also assume that electrons are confined in the metal by the surface barrier given by ${ }^{23,48}$

$$
V(z)=W+E_{F}-e U(z)-\frac{e^{2}}{16 \pi \varepsilon_{0} z},
$$

where $W$ is the work function, $U(z)$ is the electrostatic potential, and the last term represents the image charge effect ( $e$ is the elementary charge and $\varepsilon_{0}$ is the vacuum permittivity). In the field emission condition with $W$ approximately equal to $4.5 \mathrm{eV}$ (for molybdenum or tungsten) that we consider in the following, $U(z) \approx F z$ with the electric field magnitude $F$ $(>0)$ in the range of $1-5 \mathrm{GV} / \mathrm{m}$ at very close to the field emitter surface. An electron in the metal with the total energy $E$ ( $E=0$ coincides the conduction band bottom of the metal) is characterized by the wave vector $\left(k_{x}, k_{y}, k_{z}\right)$, that satisfies at zero temperature,

$$
E=\frac{\hbar^{2}}{2 m}\left(k_{x}^{2}+k_{y}^{2}+k_{z}^{2}\right) \leq E_{F} .
$$

We split $E$ to the normal energy $E_{z}=\hbar^{2} k_{z}^{2} /(2 m)$ (the kinetic energy in the $z$-direction) and the transverse energy $E_{t}=\hbar^{2}\left(k_{x}{ }^{2}+k_{y}{ }^{2}\right) /(2 m) \equiv \hbar^{2} \mathbf{k}_{\mathbf{t}}{ }^{2} /(2 m)$ (the kinetic energy in the transverse direction) where $\mathbf{k}_{\mathbf{t}}=\left(k_{x}, k_{y}\right) . V(z), E$, and $E_{z}$ are all measured relative to the conduction band bottom. $U(z)$ is taken as zero at the emitter electrical surface at $z=0$.

In the standard theory of the field emission, ${ }^{23,48-50}$ the tunneling probability $D$ through the barrier (1) calculated by the semi-classical approximation is written as

$$
D=P\left(E_{z}\right) \exp \left[-2 \int_{a}^{b}|K(z)| d z\right] \cong \exp \left[-G\left(E_{z}\right)\right],
$$

where the classical turning points $z=a$ and $b(a<b)$ are determined by $V(z)=E_{z}$, and $K(z)$ is the $z$-component of the wave vector $(z>0)$

$$
K(z)=\sqrt{\frac{2 m}{\hbar^{2}}\left\{E_{z}-V(z)\right\}} .
$$


Inside the barrier $(a<z<b), K(\mathrm{z})$ is imaginary, and it is real in the vacuum $(b<z)$. The pre-factor $P\left(E_{z}\right)$ in (3) is neglected in the following since it is of the order of 1 and, comparing to the exponent term, $G\left(E_{z}\right)$, its energy dependence can be safely neglected when we consider the energy dependence of $D .^{50}$ The field emission current density $J$ is calculated by integrating the positive $z$-component of the electron velocity $v_{z}$ (equal to $\hbar k_{z} / m$ ) times the elementary charge $e$ weighted by $D$ over the available electronic states in the solid specified by $\left(\mathbf{k}_{\mathbf{t}}, k_{z}\right)$

$$
J=2 \int_{k_{z}>0} \frac{d k_{z}}{2 \pi} \iint \frac{d \mathbf{k}_{\mathbf{t}}}{(2 \pi)^{2}} f\left(E_{z}+E_{t}\right) e^{-G\left(E_{z}\right)}\left(e v_{z}\right),
$$

where $f(E)$ is the Fermi-Dirac distribution ( $T$ is the temperature and $k_{B}$ is the Boltzmann constant)

$$
f(E)=\left[e^{\left(E-E_{F}\right) /\left(k_{B} T\right)}+1\right]^{-1} .
$$

The average transverse energy $\left\langle E_{t}\right\rangle$ that is relevant to the intrinsic transverse emittance ${ }^{44}$ is given by the following average using the integrand of (5) as the distribution function: ${ }^{23,51,52}$

$$
\left\langle E_{t}\right\rangle=\frac{2 \int_{k_{z}>0} \frac{d k_{z}}{2 \pi} \iint \frac{d \mathbf{k}_{\mathbf{t}}}{(2 \pi)^{2}} f\left(E_{z}+E_{t}\right) e^{-G\left(E_{z}\right)} E_{t}}{2 \int_{k_{z}>0} \frac{d k_{z}}{2 \pi} \iint \frac{d \mathbf{k}_{\mathbf{t}}}{(2 \pi)^{2}} f\left(E_{z}+E_{t}\right) e^{-G\left(E_{z}\right)}} .
$$

The average energies, $\langle E\rangle$ and $\left\langle E_{z}\right\rangle$, are also calculated similarly to (7). In literature, $\langle E\rangle$ and $\left\langle E_{z}\right\rangle$ are frequently defined and calculated by using the integrand of $J$ including $v_{z}$ as the distribution function. ${ }^{53}$ Both definitions give the same result in the present case with $E_{F} \gg k_{B} T$, since $v_{z}$ is replaced by the Fermi velocity and its energy dependence can be safely neglected.

The exact analytical calculations of $J$ and average energies are difficult, but highly accurate results are obtained for $E_{F} \gg k_{B} T$ by expanding $G\left(E_{z}\right)$ in Taylor series at $E_{F}$ and considering only the first order term; we write the first order expansion of $G\left(E_{z}\right)$ at $E_{F}$ as

$$
-G\left(E_{z}\right)=-G_{F}+\left(E_{z}-E_{F}\right) / d_{F} ;
$$

in which the quantities $G_{F}$ and $d_{F}$ are given in literature. ${ }^{48,50}$ We reproduce those here for completeness

$$
\begin{gathered}
G\left(E_{F}\right)=v_{F} G_{0}\left(E_{F}\right) \\
\frac{1}{d_{F}}=-\left.\frac{\partial G}{\partial E_{z}}\right|_{E_{z}=E_{F}}=-\left.t_{F} \frac{\partial G_{0}}{\partial E_{z}}\right|_{E_{z}=E_{F}}=t_{F} \frac{3}{2} \frac{b W^{1 / 2}}{F}, \\
G_{0}\left(E_{F}\right)=\frac{b W^{3 / 2}}{F} \\
v_{F} \approx 1-f_{F}+\frac{1}{6} f_{F} \ln f_{F}, \\
t_{F}=v_{F}-\frac{4}{3} f_{F} \frac{d v_{F}}{d f_{F}}
\end{gathered}
$$

$$
f_{F}=\frac{c^{2} F}{W^{2}}
$$

The constants $b$ and $c$ in the above equations are determined by fundamental constants as $b=(4 / 3) \quad\left(2 m_{0}\right)^{1 / 2} /(e \hbar)$ $\approx 6.830890 \mathrm{eV}^{-3 / 2} \mathrm{~V} \mathrm{~nm}^{-1}$ and $c=\left(e^{3} / 4 \pi \varepsilon_{0}\right)^{1 / 2} \approx 1.199985 \mathrm{eV}$ $\mathrm{V}^{-1 / 2} \mathrm{~nm}^{1 / 2}$. $^{-18}$

The quantity $d_{F}$ determines the range of the normal energies closely below the Fermi energy that contributes to the field emission current. $d_{F}$ varies proportional to $F$ and is equal to $0.13 \mathrm{eV}$ for $F=3 \mathrm{GV} / \mathrm{m}$ that is a typical $F$ value for field emission from a metal with $W$ of $\sim 4.5 \mathrm{eV}$. It is much smaller than $E_{F}(=5-10 \mathrm{eV})$, hence the approximation (8) is justified.

The field emission current density and the average energies at finite temperature are calculated as one can find in literature $^{23,52,53}$ for the temperature parameter $p \equiv k_{\mathrm{B}} T / d_{\mathrm{F}}$ to be smaller than $\sim 0.7$ (i.e., the condition in which the thermionic emission can be neglected ${ }^{53}$ )

$$
\begin{gathered}
J=\frac{e m}{2 \pi^{2} \hbar^{3}} d_{F}^{2} \exp \left(-G_{F}\right) \frac{\pi p}{\sin \pi p}, \\
\left\langle E-E_{F}\right\rangle \simeq-d_{F} \varphi(p), \\
\left\langle E_{z}-E_{F}\right\rangle \simeq-d_{F}[1+\varphi(p)], \\
\left\langle E_{t}\right\rangle=\left\langle E-E_{z}\right\rangle=d_{F} .
\end{gathered}
$$

In (16)-(17), the factor $\varphi(p)=\pi p \cot \pi p$ shows that $\left|\left\langle E-E_{F}\right\rangle\right|$ and $\left|\left\langle E_{z}-E_{F}\right\rangle\right|$ increase with the increase in the temperature, but $\left\langle E_{t}\right\rangle$ does not increase within the same approximation and the temperature range. ${ }^{23}$

\section{CALCULATION OF THE TRANSVERSE STRUCTURE OF FIELD EMISSION ELECTRON WAVE FUNCTION}

Now we calculate the wave function of the field emission electron beam. By $\psi\left(\mathbf{r}_{\mathbf{t}}, z ; \mathbf{r}_{\mathbf{t}}^{\prime}\right)$, we denote the wave function of an electron emitted from a point $\mathbf{r}_{\mathbf{t}}^{\prime}$ on the emitter surface $(z=0)$ where $\mathbf{r}_{\mathbf{t}}=(x, y)$ is the coordinate in the transverse direction. When electrons are emitted from an area $S$ on the emitter surface and they are phase-coherent, we can define the wave function $\Psi_{S}\left(\mathbf{r}_{\mathbf{t}}, z\right)$ of the field emission beam emitted from $S$. By definition, $\Psi_{S}\left(\mathbf{r}_{\mathbf{t}}, z\right)$ is given by integrating $\psi\left(\mathbf{r}_{\mathbf{t}}, z ; \mathbf{r}_{\mathbf{t}}^{\prime}\right)$ for $\mathbf{r}_{\mathbf{t}}^{\prime}$ in $S$. To consider the general case in which electrons emitted from the different points have different amplitudes and phases, we introduce the amplitude $A_{S}\left(\mathbf{r}_{\mathbf{t}}\right)$ that in general is complex. As a result, $\Psi_{S}\left(\mathbf{r}_{\mathbf{t}}, z\right)$ is written as the integral over $S$ given by

$$
\Psi_{S}\left(\mathbf{r}_{\mathbf{t}}, z\right)=\int d \mathbf{r}_{\mathbf{t}}^{\prime} A_{S}\left(\mathbf{r}_{\mathbf{t}}^{\prime}\right) \psi\left(\mathbf{r}_{\mathbf{t}}, z ; \mathbf{r}_{\mathbf{t}}^{\prime}\right) .
$$

When electrons emitted from different points within $S$ are not phase-coherent, $\Psi_{S}\left(\mathbf{r}_{\mathbf{t}}, z\right)$ is not a well-defined quantity, and the longitudinal propagation and the transverse coherence of the beam should be analyzed, e.g., by the crossspectral density function of the partial coherent beam ${ }^{29,30}$ Even in such a partially coherent case, $\psi\left(\mathbf{r}_{\mathbf{t}}, z ; \mathbf{r}_{\mathbf{t}}^{\prime}\right)$ is still a well-defined quantity. The analysis of the partially coherent beam is beyond the scope of the present work and we will 
consider only the fully coherent case in the following. Because of the translational symmetry in the transverse direction of the assumed planar cathode, $\psi\left(\mathbf{r}_{\mathbf{t}}, z ; \mathbf{r}_{\mathbf{t}}^{\prime}\right)$ is equal to $\psi\left(\mathbf{r}_{\mathbf{t}}-\mathbf{r}_{\mathbf{t}}^{\prime}, z ; 0\right)$. Therefore, in the following, we write for $\psi\left(\mathbf{r}_{\mathbf{t}}, z ; 0\right)$ as $\psi\left(\mathbf{r}_{\mathbf{t}}, z\right)$ for simplicity. We will first calculate $\psi\left(\mathbf{r}_{\mathbf{t}}, z\right)$ and consider an example of $\Psi_{S}\left(\mathbf{r}_{\mathbf{t}}, z\right)$.

When an electron wave in the metal with the wave vector $\left(\mathbf{k}_{\mathbf{t}}, k_{z}\right)$ with the positive $k_{z}\left(k_{z}>0\right)$ and the unit probability flux is incident on the surface barrier, it is mostly reflected, and a small portion of it transmits through the surface barrier with the transmission coefficient given by the square root of $D$ in Eq. (3). The wave function in the metal far from the surface is assumed to be a plane wave (Fig. 1)

$$
f_{\text {in }}+f_{r}=\frac{1}{\sqrt{v}} e^{i \mathbf{k}_{\mathbf{t}} \cdot \mathbf{r}_{\mathbf{t}}}\left[e^{i k_{z} z}+\zeta e^{-i k_{z} z}\right]
$$

with the reflection coefficient $\zeta(|\zeta| \approx 1)$. Equation $(20)$ is normalized for the unit electron flux and $v$ is the velocity of the electron. When the transverse momentum $\hbar k_{t}$ is much smaller than the momentum $\hbar k_{z}$ in the $\mathrm{z}$-direction as in the presently considered case, $v$ is approximately equal to the $z$-component of the velocity $v_{\mathrm{z}}=\hbar k_{z} / m$. In (20), we omitted the contribution of the crystal lattice on the electron wave function in the solid by assuming the free-electron model of metal. ${ }^{23,47-49,51}$

In (20), we included the transverse plane wave $\exp \left(\mathrm{ik}_{\mathbf{t}} \cdot \mathbf{r}_{\mathbf{t}}\right)$ with the transverse wave vector $\mathbf{k}_{\mathbf{t}}$. In the vacuum side $(z \gg b)$, the $z$-dependence of the wave function propagating in the positive $z$-direction is obtained by the WentzelKramers-Brillouin (WKB) solution. ${ }^{54}$ Here, we extend it by

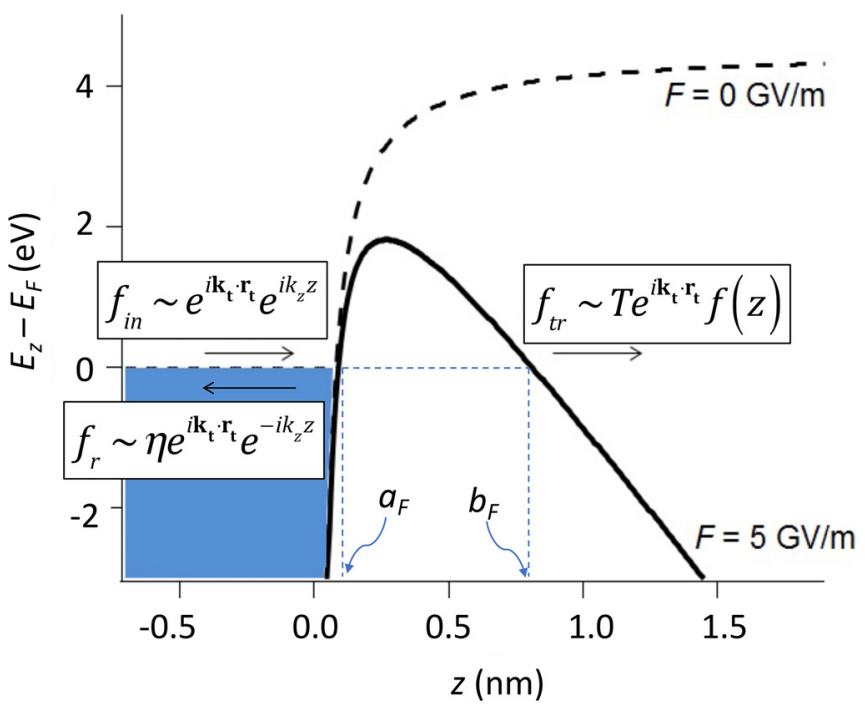

FIG. 1. The schematic energy diagram of the metal surface under the strong electric field $F$. The incident wave $f_{\text {in }}$ is mostly reflected to $f_{r}$ (with the reflection coefficient $\eta \sim 1$ ) but a small fraction tunnels into $f_{\text {tr }}$ through the barrier (with the small transmission coefficient $T \ll 1$ ). Note that $\eta$ and $T$ are not to be confused with the reflection probability $|\eta|^{2}$ and the transmission probability $D=|T|^{2}$. When the tunneling electron was originated from an electronic state with the momentum $\left(\hbar \mathbf{k}_{\mathbf{t}}, \hbar k_{z}\right)$ in the solid and the transverse momentum $\hbar \mathbf{k}_{\mathbf{t}}$ is finite, all the wave functions are proportional to the plane wave $\exp \left(\mathbf{i k}_{\mathbf{t}} \cdot \mathbf{r}_{\mathbf{t}}\right)$ because of the conservation of the transverse momentum ( $\mathbf{r}_{\mathbf{t}}$ is the transverse coordinate), see the discussion related to (21-22). The barrier of the metal surface under the field emission condition at the surface electric field $F$ of $5 \mathrm{GV} / \mathrm{m}$ (solid line) is calculated for the work function of $4.5 \mathrm{eV}$. At $z=a_{F}$ and $b_{F}$, the surface barrier is at the Fermi energy. The dashed line shows the case when $F=0 \mathrm{~V} / \mathrm{m}$. multiplying the transverse plane wave $\exp \left(\mathbf{i k}_{\mathbf{t}} \cdot \mathbf{r}_{\mathbf{t}}\right)$ with the identical transverse wave vector $\mathbf{k}_{\mathbf{t}}$ specified in the wave function inside the metal (20) as

$$
f_{t r}\left(\mathbf{r}_{\mathbf{t}}, z\right)=\sqrt{D} \frac{e^{i \mathbf{k}_{\mathbf{t}} \cdot \mathbf{r}_{\mathbf{t}}}}{\sqrt{v(z)}} \exp \left[i \int_{b}^{z} K(z) d z-\frac{i \pi}{4}\right] .
$$

The velocity $v(z)$ is well-approximated by its $z$-component at large $z(\gg b)$,

$$
v(z)=\left.\frac{\hbar}{m} \sqrt{k_{x}^{2}+k_{y}^{2}+K^{2}(z)} \approx \frac{\hbar}{m} K(z)\right|_{b \ll z} .
$$

$K(z)$ in (21), (22) is given in (4). We included the transverse plane wave in (21) because the transverse momentum $\hbar \mathbf{k}_{\mathbf{t}}$ conserves upon tunneling through the surface barrier at the planar metal surface. ${ }^{52}$ This is not the case for the momentum in the $z$-direction because the tunneling barrier breaks the translational symmetry of the system in this direction. Still the probability flux in the $z$-direction is conserved with (21). ${ }^{54,55}$ The lower integration limit $z=b$ in the exponent of (21) is determined here by the relation $e U(b) \cong W+E_{F}-E_{z}$ by neglecting the image potential energy in (1). This approximation is justified since in the case of $U(z) \cong F z$ at $z \sim b$ with $F=1-5 \mathrm{GV} / \mathrm{m}$ and $W \sim 4.5 \mathrm{eV}$, the image potential energy contributes at most $10 \%$ near $z \sim b$ and becomes smaller at $z \gg b$. When $F$ is constant and the image potential energy is negligible, the $z$-dependence of the vacuum-side wave function is given by the Airy functions, ${ }^{46,55}$ and its asymptotic form agrees with (21). The phase $\pi / 4$ included in (21) is because of the phase of the asymptotic form of the Airy function.

With these preparations, now we calculate the wave function $\psi\left(\mathbf{r}_{\mathbf{t}}, z\right)$ for a field emission electron averaged over the available electronic states weighted by the transmission probability. For that we integrate (21) given for a specific $\left(\mathbf{k}_{\mathbf{t}}, k_{z}\right)$ in the solid over $\left(\mathbf{k}_{\mathbf{t}}, k_{z}\right)$ weighted by the Fermi-Dirac distribution and the transmission coefficient $\sqrt{ } D$ by the otherwise same method to calculate (5) and (7)

$$
\psi=\frac{2 \int_{k_{z}>0} \frac{d k_{z}}{2 \pi} \iint \frac{\left.d \mathbf{r}_{\mathbf{t}}, z\right)}{(2 \pi)^{2}} f\left(E_{\mathbf{k}_{\mathbf{t}}, k_{z}}\right) \sqrt{D} \frac{e^{i \mathbf{k}_{\mathbf{t}} \cdot \mathbf{r}_{\mathbf{t}}}}{\sqrt{v}} \exp \left[i \int_{b}^{z} K(z) d z-\frac{i \pi}{4}\right]}{2 \int_{k_{z}>0} \frac{d k_{z}}{2 \pi} \iint \frac{d \mathbf{k}_{\mathbf{t}}}{(2 \pi)^{2}} f\left(E_{\mathbf{k}_{\mathbf{t}}, k_{z}}\right) \sqrt{D}} .
$$

For simplicity, we again assume the temperature $T=0$. The integration of the denominator of (23) is straightforward and is equal to

$$
\begin{gathered}
\frac{1}{4 \pi^{2}} \sqrt{\frac{2 m}{\hbar^{2} E_{F}}} e^{-G_{F} / 2} \int_{0}^{\infty} k_{t} d k_{t} \int_{E_{t}}^{\infty} d \varepsilon e^{-\varepsilon /\left(2 d_{F}\right)} \\
=\frac{1}{4 \pi^{2}} \sqrt{\frac{2 m}{\hbar^{2} E_{F}}} e^{-G_{F} / 2} \frac{1}{2}\left(\frac{2 m}{\hbar^{2}}\right)\left(2 d_{F}\right)^{2},
\end{gathered}
$$


where we defined the integration variable $\varepsilon=E_{F}-E_{z}$. To integrate the numerator of (23), we first approximate the $E_{z^{-}}$ dependence by the first order term near $E_{F}$

$$
\begin{gathered}
\int_{b}^{z} K(z) d z \simeq \alpha(z)+\beta(z)\left(E_{z}-E_{F}\right), \\
\alpha(z)=\int_{b_{F}}^{z} K_{F}(z) d z \\
K_{F}(z)=\sqrt{\frac{2 m}{\hbar^{2}}\{e U(z)-W\}} \\
\beta(z)=\left.\frac{\partial}{\partial E_{z}} \int_{b}^{z} K(z) d z\right|_{E_{z}=E_{F}}=-\frac{\partial}{\partial W} \alpha(z),
\end{gathered}
$$

where $b_{F}$ is determined by $e U\left(b_{F}\right)=W$. By using these definitions, the integration of the numerator of (23) is calculated as follows ( $J_{O}$ is the zeroth order Bessel function)

$$
\begin{aligned}
\frac{1}{4 \pi^{2}} & \sqrt{\frac{2 m}{\hbar^{2} E_{F}}} e^{-G_{F} / 2} \frac{e^{i(\alpha-\pi / 4)}}{\sqrt{v_{F}}} \int_{0}^{\infty} k_{t} d k_{t} J_{0}\left(k_{t} r_{t}\right) \int_{E_{t}}^{\infty} d \varepsilon e^{-\gamma \varepsilon} \\
= & \frac{1}{4 \pi^{2}} \sqrt{\frac{2 m}{\hbar^{2} E_{F}}} e^{-G_{F} / 2} \frac{e^{i(\alpha-\pi / 4)}}{\sqrt{v_{F}}} \\
& \times \frac{1}{\gamma} \int_{0}^{\infty} k_{t} d k_{t} J_{0}\left(k_{t} r_{t}\right) e^{-\gamma \hbar^{2} k_{t}^{2} /(2 m)} \\
= & \frac{1}{4 \pi^{2}} \sqrt{\frac{2 m}{\hbar^{2} E_{F}}} e^{-G_{F} / 2} \frac{e^{i(\alpha-\pi / 4)}}{\sqrt{v_{F}}} \frac{1}{\gamma} \frac{1}{2}\left(\frac{2 m}{\gamma \hbar^{2}}\right) \\
& \times \exp \left[-\frac{1}{4}\left(\frac{2 m}{\gamma \hbar^{2}}\right) r_{t}^{2}\right] .
\end{aligned}
$$

In (29), we defined

$$
\gamma(z) \equiv \frac{1}{2 d_{F}}+i \beta(z) .
$$

We also used the following formula ${ }^{56,57}$ to perform the integral in the last line:

$$
\int_{0}^{\infty} d x e^{-a^{2} x^{2}} x J_{0}(b x)=\frac{1}{2 a^{2}} e^{-b^{2} /\left(4 a^{2}\right)}
$$

with $a^{2}$ taken to be equal to $\gamma \hbar^{2} /(2 m)$, and $b$ to be equal to $r_{t}$. As a result, we obtain

$$
\psi\left(\mathbf{r}_{\mathbf{t}}, z\right)=\frac{e^{i(\alpha-\pi / 4)}}{\sqrt{v_{F}}} \frac{1}{\left(2 d_{F} \gamma\right)^{2}} \exp \left[-\frac{m}{2 \gamma \hbar^{2}} r_{t}^{2}\right]
$$

By separating the real part and the imaginary part of $\gamma(z)$

$$
\frac{1}{\gamma(z)}=2 d_{F}\left[\frac{1}{1+4 d_{F}^{2} \beta^{2}(z)}-\frac{2 i d_{F} \beta(z)}{1+4 d_{F}^{2} \beta^{2}(z)}\right],
$$

we can rewrite (32) in a form similar to the optical Gaussian beam by using the transverse rms beam radius $\sigma$ and the wave front radius of curvature $R$

$$
\begin{gathered}
\psi\left(\mathbf{r}_{t}, z\right)=\frac{e^{i(\alpha-\pi / 4-2 \xi)}}{\sqrt{v_{F}}} \frac{\sigma_{0}^{2}}{\sigma^{2}} \exp \left(-\frac{r_{t}^{2}}{2 \sigma^{2}}\right) \exp \left(i \frac{K_{F} r_{t}^{2}}{2 R}\right), \\
\sigma^{2}(z)=\sigma_{0}^{2}+\frac{1}{\sigma_{0}^{2}}\left\{\frac{\hbar^{2} \beta(z)}{m}\right\}^{2}, \\
R(z)=K_{F}(z)\left[\frac{\hbar^{2}}{m} \beta(z)+\frac{\sigma_{0}^{4}}{\hbar^{2} \beta(z) / m}\right], \\
\sigma_{0}^{2}=\frac{\hbar^{2}}{2 m d_{F}}, \\
\xi=\tan ^{-1}\left(\frac{1}{\sigma_{0}^{2}} \frac{\hbar^{2} \beta}{m}\right) \\
v_{F}(z)=\frac{\hbar}{m} K_{F}(z) .
\end{gathered}
$$

Since $\beta(z)$ goes to zero as $z$ approaches the metal surface, $\sigma_{0}$ in (37) determines the minimal transverse wave function spread at the metal surface. By utilizing the cylindrical symmetry of the system along the beam axis and noting the fact that for emitted electrons

$$
\left\langle E_{t}\right\rangle=\left\langle\frac{1}{2 m}\left(\hbar^{2} k_{x}^{2}+\hbar^{2} k_{y}^{2}\right)\right\rangle=\frac{1}{m}\left\langle\hbar^{2} k_{x}^{2}\right\rangle=d_{F},
$$

where $k_{x}=k_{y}$ by the assumed cylindrical symmetry, we can rewrite $\sigma_{0}$ as

$$
\sigma_{0}=\frac{\hbar}{\sqrt{2\left\langle\hbar^{2} k_{x}^{2}\right\rangle}}
$$

Equation (41) shows that the minimal transverse spatial spread of the wave function of the field emission electron is determined by the finite transverse momentum spread, which is given by the fact that only the electrons in the metal with energies within $d_{F}$ of the Fermi level have significant amplitude outside the metal. This is expected from the uncertainty relationship between the transverse position and the transverse momentum. When $F=3 \mathrm{GV} / \mathrm{m}$ and $d_{F}=0.13 \mathrm{eV}, \sigma_{0}=0.54 \mathrm{~nm}$ (for $F=1-5 \mathrm{GV} / \mathrm{m}, \sigma_{0}=0.94-0.42 \mathrm{~nm}$, respectively).

When a field emission electron beam is produced from a finite and fully coherent area $S$, the total wave function of the beam can be calculated by substituting (32) or (34) into (19). As an example, we consider $A_{S}(\mathbf{r})=\exp \left[-\mathbf{r}^{2} /\left(2 a_{s}^{2}\right)\right]$ where $a_{s}$ is the rms radius of $S$. We found that $\Psi_{S}\left(\mathbf{r}_{\mathbf{t}}, z\right)$ is given by

$$
\begin{aligned}
\Psi_{S}(\mathbf{r}, z)= & \frac{e^{i(\alpha-\pi / 4-2 \xi)}}{\sqrt{v_{F}}} \frac{\sigma_{0}^{2}}{\sigma^{2}} \frac{a^{2}\left(\sigma_{0}^{2}+i \hbar^{2} \beta / m\right)}{a^{2}+\sigma_{0}^{2}+i \hbar^{2} \beta / m} \\
& \times \exp \left(-\frac{\mathbf{r}^{2}}{2 \sigma_{a}^{2}}\right) \exp \left(i \frac{K_{F} \mathbf{r}^{2}}{2 R_{a}}\right), \\
\sigma_{a}^{2}(z)= & a_{s}^{2}+\sigma_{0}^{2}+\frac{1}{a_{s}^{2}+\sigma_{0}^{2}}\left\{\frac{\hbar^{2} \beta(z)}{m}\right\}^{2}, \\
R_{a}(z)= & K_{F}(z)\left[\frac{\hbar^{2}}{m} \beta(z)+\frac{\left(a_{s}^{2}+\sigma_{0}^{2}\right)^{2}}{\hbar^{2} \beta(z) / m}\right],
\end{aligned}
$$


that is, the transverse variation of the wave function is given by replacing $\sigma_{0}^{2}$ with $\left(\sigma_{0}^{2}+a_{s}^{2}\right)$.

We focused in this calculation the analysis of the transverse structure that is relevant to the transverse spatial coherence characteristics of the beam (emitted from a planar cathode). Therefore, we neglect the influence of the finite longitudinal coherence of the beam that is normally of less influence on the transverse spatial coherence. ${ }^{28,58}$ In the above calculation, this was implied by assuming that the longitudinal phase propagation is given by (27) without finite longitudinal energy spread.

\section{DISCUSSIONS}

\section{A. Numerical example 1: Constant $\boldsymbol{F}$ case}

Now we examine the implication of the thus obtained wave function (32), (34), and (42) with some examples. First, we consider the case in which $U(z)=F z$ with a constant $F . K_{F}(z), \alpha(z)$, and $\beta(z)$ are written as follows (we omit $b_{F}=W /(e F)$ by shifting the origin of $z$ to $\left.b_{F}\right)$

$$
\begin{gathered}
K_{F}(z)=\sqrt{\frac{2 m e F}{\hbar^{2}}} z, \\
\alpha(z)=\frac{2}{3} \sqrt{\frac{2 m e F}{\hbar^{2}}} z^{3 / 2},
\end{gathered}
$$

$$
\begin{gathered}
\beta(z)=\frac{1}{e F} \sqrt{\frac{2 m e F}{\hbar^{2}}} z, \\
\sigma(z)=\sqrt{\sigma_{0}^{2}+\frac{1}{\sigma_{0}^{2}} \frac{2 \hbar^{2}}{m e F} z,} \\
R(z)=2 z+\frac{m e F}{\hbar^{2}} \sigma_{0}^{4} .
\end{gathered}
$$

In Fig. 2, we show $\alpha(z), \beta(z), \sigma(z), R(z)$, the real part of the wave function near the emitter $(\mathrm{z}<12 \mathrm{~nm})$, and the variation of the norm of the wave function in a larger z-range $(<100 \mathrm{~nm})$ in the case of $F=3 \mathrm{GV} / \mathrm{m}$ and $W=4.5 \mathrm{eV}$ (hence $d_{F}=0.13 \mathrm{eV}$ with $b_{F}=W / e F=1.5 \mathrm{~nm}$ ). Figure 2(a) shows that $\left|\beta(z)\left\langle E_{z}-E_{F}\right\rangle\right| \sim \beta(z) d_{F}$ is smaller than $\alpha(z)$, hence the assumption implied in the expansion in (25) is satisfied. Figure 2(b) shows that in this constant $F$ case, $R(z)$ varies as $2 z$ and $\sigma(z)$ increases as $\sqrt{ } z$. We note that $d_{F}$ (and $1 / \sigma_{0}{ }^{2}$ ) are proportional to $F$, hence the second term of (48) is independent of $F$. By substituting (10) and (37) into (48),

$$
\sigma(z) \approx \sqrt{\sigma_{0}^{2}+\frac{2 z}{\left(2 m W / \hbar^{2}\right)^{1 / 2}}} \rightarrow \sqrt{\frac{\lambda_{W}}{\pi} z} \quad\left(\sigma_{0}^{2} / \lambda_{W} \ll z\right),
$$

where the length $\lambda_{w}$ in (50) is defined by the work function
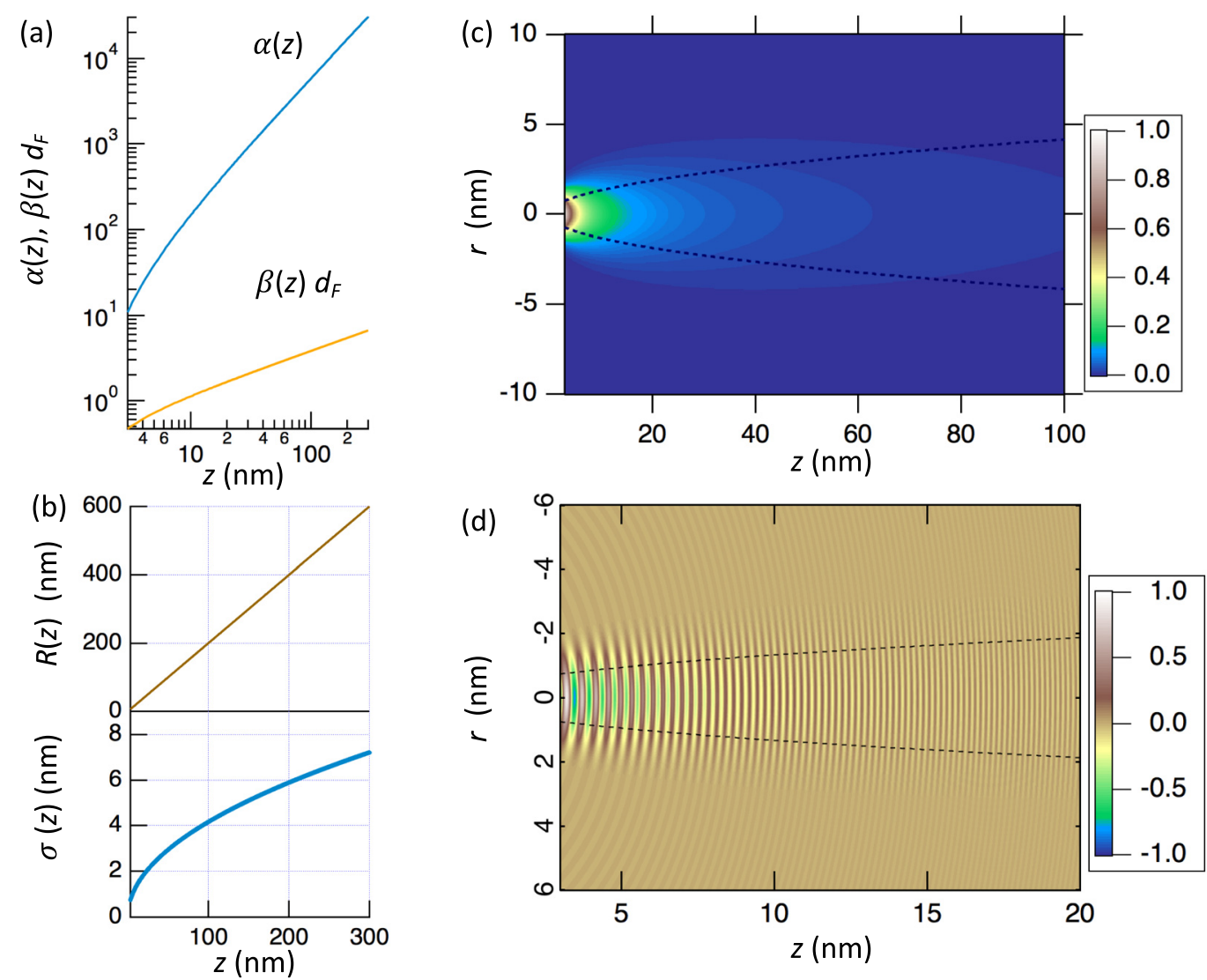

FIG. 2. Numerical example 1 of the wave function of field emission electron beam: the case of a constant electric field $F=3 \mathrm{GV} / \mathrm{m}$. Despite the assumption that the cathode is a point emitter, the wave function exhibits the finite width as determined by the average transverse energy. (a) the longitudinal phase $\alpha(\mathrm{z})$ and the transverse phase $\beta(z)$. Note that $\beta(z)$ is multiplied by $d_{F}=0.13 \mathrm{eV}$. (b) The variation of the rms transverse spread $\sigma(z)$ and the radius of the curvature $R(z)$. (c) The norm of the wave function (normalized by the maximum value). The broken curves indicate $r= \pm \sigma(z)$. (d) The real part of the wave function in the range of $z=3-12 \mathrm{~nm}$ and $r=-6$ to $6 \mathrm{~nm}$. The amplitude is normalized by the maximum value. 


$$
\lambda_{W}=\frac{2 \pi}{\sqrt{2 m W / \hbar^{2}}}
$$

$\lambda_{w}$ is equal to $0.6 \mathrm{~nm}$ for $W=4.5 \mathrm{eV}$, hence the large $z$ limit in (50) is satisfied except within $\sim 1 \mathrm{~nm}$ from the cathode surface.

Figure 2(c) shows that the rms transverse radius $\sigma(z)$ of $\psi\left(\mathbf{r}_{\mathbf{t}}, z\right)$ is equal to $\sigma_{0}(\sim 0.5 \mathrm{~nm})$ near the emitter surface. $\sigma(z)$ increases slowly with the increase in $z$ (the curves indicate $\sigma(z)$ ), and the electron wavelength rapidly decreases at the same time because of the acceleration. The oscillation amplitude decreases with the propagation because of the increase in the rms transverse spread [Fig. 2(d)].

Let us compare the present problem with a free space propagation of a finite energy electron emitted from a point. This can be considered, e.g., a fixed energy electron plane wave is incident on a wall with an infinitely small hole. The wave function on the opposite side of the wall is a spherical wave. The wave front radius of the curvature is given by the propagation distance from the hole and the wave spreads over the half sphere. Comparing to that, in the case of the propagation of an electron emitted from a point at zero energy and accelerated in a constant $F$ as calculated here, the transverse spread of the electron wave function is largely restricted even though the radius of the curvature of the wave front increases proportionally to the propagation distance.

These characteristics were also predicted by the recent analysis of the transverse structure of the field emission electron wave function reported in Ref. 46. They solved the Schrödinger's equation for electron emission from a small area of planar electrodes under a uniform electric field with non-zero transverse momentum. Different from our approach, they calculated the transverse structure of the wave function by mathematically specifying an emission area at the metal surface. When the electric field is constant and the emission on the cathode surface varies as a Gaussian $\exp \left[-\mathbf{r}^{2} /\left(2 a^{2}\right)\right]$, they obtained a wave function that varies in the transverse direction as

$$
|\Psi|^{2} \sim \exp \left[-\frac{r_{t}^{2}}{2 z}\left\{\left(\frac{2 m W}{\hbar^{2}}\right)^{1 / 2}+\frac{a^{2} e m F}{\hbar^{2}}\right\}\right] .
$$

For comparison, we write the probability distribution from (42) in the same constant $F$ situation at large $z$ as

$$
\left|\Psi_{S}\left(\mathbf{r}_{t}, z\right)\right|^{2} \propto \exp \left(-\frac{r_{t}^{2}}{\sigma^{2}}\right) \rightarrow \exp \left[-\frac{r_{t}^{2}}{2 z}\left(a_{s}^{2}+\sigma_{0}^{2}\right) \frac{e m F}{\hbar^{2}}\right] .
$$

This is different from (52). In (53), in the limit of zero $a_{s}$, it is $\sigma_{0}$ that determines the $z$-dependence of the transverse wave function spread. When $a_{s}$ is much larger than $\sigma_{0}$, it is $a_{s}$ that determines the $z$-dependence of the transverse wave function spread without the first term in the argument of (52). We consider that the difference of (52) from (53) originated from the neglect of the restriction on the possible form of the solution of the Schrödinger equation by the available electronic states in solid weighted by the transmission probability through the surface barrier.

\section{B. Numerical example 2: The case of the field enhancement and the rapidly decaying electric field away from the emitter}

In actual field emitters, the high electric field at the tip apexes is achieved with the help of the field enhancement, and the electric field decreases rapidly as the electron propagates away from the emitter surface. ${ }^{50}$ We therefore study next a case when the electric field decays away from the emitter surface. As an example, we consider the following model potential (taking $z=0$ as the surface):

$$
U(z)=U_{0}\left(1-\frac{1}{1+z / r_{t i p}}\right) .
$$

This is exact (along the $z$-axis) when the emitter tip is a sphere with the radius of $r_{t i p}$ and the anode is a concentric sphere with the radius of $r_{a}\left(r_{a} \gg r_{t i p}\right)$. For the calculation of $d_{F}$, we assume in addition that the electric field at the tip is given by $U_{o} /\left(k r_{\text {tip }}\right)$ with $k \sim 5$ (but not for $K_{F}(z), \alpha(z)$, and $\beta(z)$ ). The factor $k \sim 5$ is normally assumed to take into account the partial screening due to the shank of needleshaped etched-wire emitters. ${ }^{59}$ Despite these assumptions, we still assume a planar cathode and the transverse variation of the electrostatic potential and the associated magnification of the wave function is not considered in the following.

In Fig. 3, we display $K_{F}(z)$ [Fig. 3(a)], $R(z)$ and $\sigma(z)$ [Fig. 3(b), solid curves] calculated by assuming (54) with $r_{\text {tip }}=5 \mathrm{~nm}$ and $U_{O}=75 \mathrm{~V}$ (so that $U_{O} /\left(k r_{\text {tip }}\right)=3 \mathrm{GV} / \mathrm{m}$ ). When $z$ is increased, $K_{F}(z)$ quickly converges (already at $\mathrm{z}$ $\sim 10 \mathrm{~nm}$ ) to the value $K_{F}$ determined by the bias potential $\left(=e U_{0}\right)$. The field emission electrons acquire more than $1 \mathrm{eV}$ of the longitudinal energy after $\sim 1 \mathrm{~nm}$ propagation. Since this is much larger than $E_{t}$, the field emission electrons form a beam similarly to the case of Fig. 2 .

\section{Behavior of the wave function near the emitter surface}

Considering the fact that the Fermi wavelength is $0.4-0.6 \mathrm{~nm}$ for metals with the Fermi energy of 5-10 eV discussed in the present work, the planar-cathode assumption is reasonable for field emitters with $r_{\text {tip }}$ larger than a few nanometers for the $z$-range near the emitter-apex where the minimal transverse spread $\sigma_{0}$ has most significant impact. We discuss the behavior of the wave functions at large $z$ and its comparison with the several situations in Sec. IV D.

Near the emitter surface, the limited transverse spread of the wave function may contribute to the few nanometers spatial resolution in the near field emission scanning electron microscopy experiment, ${ }^{47,60-62}$ in which a sharp tip is scanned over a sample within a few nanometer distance and electrical potential is applied at the same time to induce field emission from the tip or from the sample. The information on the sample topography down to atomic steps was obtained either by the emission current or by collecting secondary electron. Zanin et al. reported that the spatial resolution of $\sim 1 \mathrm{~nm}$ was achieved for monatomic Fe islands on W(110) surfaces when a tungsten tip with the tip apex radius of curvature in the range of $2-7.5 \mathrm{~nm}$ was scanned over the sample 

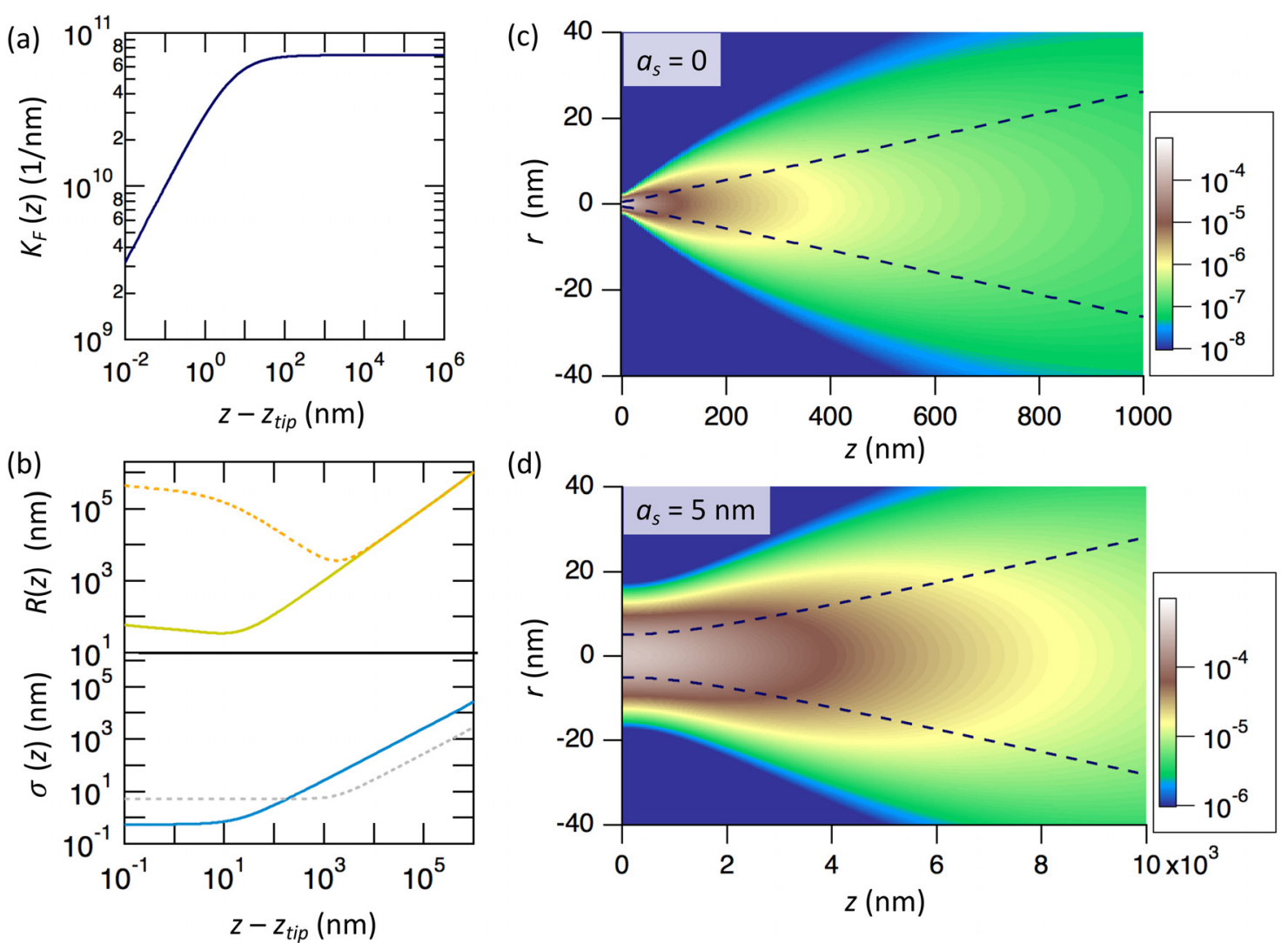

FIG. 3. Numerical example 2 of the wave function of field emission electron beam: the case when the electric field is rapidly decreasing from $F=3 \mathrm{GV} / \mathrm{m}$ from the field emitter surface with the increase in the distance [see Eq. (54)]. (a) the $z$-dependence of the wave vector. (b) the z-dependence of the rms transverse spread $\sigma(z)$ and the radius of the curvature $R(z)$ in the case when the emission originates from a "point" (solid curves) and when the electrons are emitted from a finite area with the rms radius as $=5 \mathrm{~nm}$ (broken curves). (c) and (d) show the corresponding evolution of the wave function (norm), when $a_{s}=0$ (c) and $a_{s}=5 \mathrm{~nm}(\mathrm{~d})$. The color scale for the amplitudes is also shown.

at $\sim 7 \mathrm{~nm}$-distance. ${ }^{60}$ The fact that the estimated spatial resolution ${ }^{60}$ is larger than the observation motivated the examination of the effect of the non-spherical potential distribution around the tip and the resulting lens-less focusing. ${ }^{63} \mathrm{We}$ consider that, in addition to those effects, the fact that the transverse spread of the wave function is strongly suppressed for the first few nanometers as illustrated in Figs. 2 and 3 may also contribute to the observed $\sim 1 \mathrm{~nm}$-spatial-resolution of the experiment by Zanin et al. in Ref. 62 .

When a metal nanotip is excited by a few-cycle near infrared laser pulse with a few $\mathrm{GV} / \mathrm{m}$ electric field amplitude, the electron emission exhibits a rich variety of strong-field physics at the nanotip surface. The quivering motion of the emitted electrons at the nanotip surface results in the carrier-envelopephase-sensitive electron interference and re-scattering at the tip surface $^{24}$ but only when the quivering amplitude is within a few nanometers from the nanotip surface where the optical field amplitude is enhanced by the field enhancement or the lightning rod effect. ${ }^{25,27}$ To describe the ultrafast electron dynamics at and near the nanotip surfaces, it is apparent that the present theory needs to be extended including the finite longitudinal energy bandwidth. However, it is likely that the consideration of the finite average transverse energy is important for such theory. Similar to the presently considered steady state case, the finite average transverse energy will limit the transverse spread during the longitudinal, quivering motion and acceleration, thus preventing the dilution of the wave function amplitude in the longitudinal direction. In Ref. 26, Wachter et al. showed that simulation based on the one-dimensional time-dependent density functional theory ${ }^{26}$ excellently explains the observations for tungsten nanotip experiment under 6.5-fslaser pulse excitation with the wavelength of $800 \mathrm{~nm}$. To check the validity of their one-dimensional theory, they evaluated the transverse spread of electrons upon re-scattering at the tip surface by a Monte-Carlo simulation in the case of the finite transverse momentum spread. They found that the transverse spread was limited to $0.8 \mathrm{~nm}$ in diameter with the average transverse momentum of 0.1 in atomic unit. ${ }^{26}$ The predicted transverse spread was much smaller than the tip radius $(\sim 6 \mathrm{~nm})$ in the experiment they considered, hence their one-dimensional theory was justified. We point out here that their assumption corresponds to $E_{t}$ of $\sim 0.3 \mathrm{eV}$ (with the work function of $4.35 \mathrm{eV}$ for tungsten (310) surface and the laser field amplitude of $\sim 8 \mathrm{GV} / \mathrm{m}$ ) and their prediction for the $0.8 \mathrm{~nm}$-spread agrees well with $2 \sigma_{0}$ calculated from (10) and (37) with this $E_{t}$-value.

\section{The propagation of the wave function at large distance from the emitter surface}

Now we discuss the behavior of the wave function (42) when we assumed the potential (54) that quickly decays away as the distance from the emitter surface increases. When the potential varies only along the z-direction as assumed by (54), the propagation of the wave function may 
bear a similarity to the corresponding classical situation. When an electron is emitted at $\left(\mathbf{r}_{\mathbf{t}}, z\right)=(0, \mathrm{~b})$ with the zero longitudinal velocity $\left(v_{z}=0\right)$ and the finite transverse velocity $\left(v_{t}=\left(2 d_{F} / m\right)^{1 / 2}\right)$, the orbit as determined by the potential (54) can be analytically integrated and converges to the following:

$$
\frac{r_{t}}{z} \rightarrow \sqrt{\frac{d_{F}}{e U_{0}}}
$$

To compare (55) with the value expected from the wave function (42), we calculate the average of $r_{t}^{2}$

$$
\left\langle r_{t}^{2}\right\rangle=\frac{\iint d x d y\left(x^{2}+y^{2}\right)\left|\Psi_{s}(x, y, z)\right|^{2}}{\iint d x d y\left|\Psi_{s}(x, y, z)\right|^{2}}=\sigma_{a}^{2}(z),
$$

where $\sigma_{\mathrm{a}}(z)$ is given by (43). At large $z$, we find

$$
\frac{\sqrt{\left\langle r_{t}^{2}\right\rangle}}{z}=\frac{\sigma_{a}(z)}{z} \rightarrow \sqrt{\frac{\sigma_{0}^{2}}{a_{s}^{2}+\sigma_{0}^{2}}} \sqrt{\frac{d_{F}}{e U_{0}-W}} .
$$

From the comparison of (55) and (57), we find two things. First, when $a_{s}=0$, the asymptotic transverse spread of the wave function (57) agrees to the one expected from the classical calculation (55) in the present, planar acceleration case (note that $e U_{0} \gg W$ ). Second, when $a_{s}$ is finite, the asymptotic transverse spread of the wave function is smaller than the zero- $a_{s}$ case. The corresponding transverse variation of the wave function $\exp \left[-\left(K_{F} a_{s} r_{t} / z\right)^{2}\right]$ is qualitatively different from the constant field case (50). Mathematically, the reduction of the transverse spread is a consequence of the low-spatial-frequency (=transverse wave vector) filtering effect of the integration of the wave over the finite area in (19).

In Figs. 3(c) and 3(d), we show the propagation of the wave functions in the case of $a_{s}=0$ and $5 \mathrm{~nm}$, respectively. Note the 10 times longer $z$-axis in the latter that gives approximately the same transverse spread as the zero- $a_{s}$ case, in agreement with (57) from $\sigma_{0} / a_{s} \sim 1 / 10$ with $\sigma_{0} \sim 0.5 \mathrm{~nm}$. If the electrons in the solid were completely incoherent over the source area, the resulting transverse spread is given by the zero- $a_{s}$ value. Therefore, this is a consequence of the interference of the waves emitted from the coherent source area, and this is not anticipated classically. Such a narrow electron beam spread in the case of coherent field emission source was also illustrated recently by Latychevskaia. ${ }^{58}$ The result here also suggests that, when the phase coherence length of the electrons in the solid reaches the source size, the assumption of the complete incoherence over the cathode area, that is often assumed in literature, ${ }^{28}$ may cause a substantial error for evaluating the electron source size from the interference experiments. ${ }^{17}$

The linear variation of $R(z)$ and $\sigma(z)$ with $z$ at large $z$ is same as the propagation of an optical Gaussian beam. ${ }^{64}$ This is also compatible with the propagation of a wave in free space: ${ }^{65,66}$ when a plane wave $\exp (i K z)$ is incident perpendicular to an aperture on the $(\xi, \eta)$-plane at $z=0$ with the transmission function given by $\exp \left[-\left(\xi^{2}+\eta^{2}\right) /\left(2 a^{2}\right)\right]$, the wave amplitude distribution $\Psi(X, Y)$ on the $(X, Y)$-plane parallel to the $(\xi, \eta)$-plane and located at $z(z \gg a$. More precisely, $z \gg\left(K a^{4} / 8\right)^{1 / 3}$, see Ref. 65) is given by the Fresnel integral

$$
\begin{aligned}
\Psi(X, Y)= & \frac{-i K e^{i K z}}{2 \pi z} \iint d \xi d \eta \exp \left[-\frac{\xi^{2}+\eta^{2}}{2 a^{2}}\right] \\
& \times \exp \left[\frac{i K}{2 z}\left\{(\xi-X)^{2}+(\eta-Y)^{2}\right\}\right] .
\end{aligned}
$$

After a straightforward integration, this is written as the Gaussian form

$$
\begin{aligned}
\Psi(X, Y)= & e^{i K z} \frac{-i K a^{2} / z}{1-i K a^{2} / z} \exp \left[-\frac{1}{2 \Sigma^{2}}\left(X^{2}+Y^{2}\right)\right] \\
& \times \exp \left[\frac{i K}{2 \Lambda}\left(X^{2}+Y^{2}\right)\right]
\end{aligned}
$$

where the transverse width function $\Sigma(z)$ and the wave front curvature function $\Lambda(z)$ are given by the following:

$$
\begin{gathered}
\Sigma(z)=\left(\frac{K^{2} a^{2} / z^{2}}{1+K^{2} a^{4} / z^{2}}\right)^{-1 / 2} \rightarrow \frac{z}{K a} . \\
\Lambda(z)=z\left(1+K^{2} a^{4} / z^{2}\right) \rightarrow z .
\end{gathered}
$$

In (59) and (60), their large- $z$ forms are also shown. These are identical to the large- $z$ limit of (43) and (44) of the wave function $\Psi_{S}\left(\mathbf{r}_{\mathbf{t}}, z\right)$.

\section{E. Comparison with the classical electron optics analysis and the influence of the electron coherence}

Around the tip apex of realistic field emitters, the acceleration potential has a transverse component, therefore electrons are deflected away from the $z$-axis by the transverse electric field. In this case, the angle of the asymptotic classical electron orbit from the $z$-axis becomes larger than the planar case given above (55). In the case that the emitter is a sphere with the radius of $r_{t i p}$, the anode is a concentric sphere with the radius $r_{a}\left(r_{a} \gg r_{t i p}\right)$, the field at the emitter tip is $F_{t i p}$ $\left(=e U_{0} / r_{\text {tip }}\right.$ in the present case that the emitter is a sphere), and the transverse kinetic energy is $\left\langle E_{t}\right\rangle$, the deflection angle $\omega$ (or so-called blurring fall-off half-angle) ${ }^{13,14,21-23}$ is given by

$$
\omega=2 \sqrt{\frac{\left\langle E_{t}\right\rangle}{e F_{t i p} r_{t i p}}} .
$$

Comparing with (55) calculated in the planar acceleration potential case, the fact that $\omega$ given by (61) is a factor of 2 larger (55) is ascribed to the transverse acceleration around the curved emitter surface. The actual value of $\omega$ depends on the field emitter shape and the anode configuration. Also, the energy $\left(e F_{\text {tip }} r_{\text {tip }}\right)$ in (61) is equal to the kinetic energy of an electron $U_{\infty}$ at infinity only in the case of the spherical 
emitter in the concentric spherical potential. In general, $\left(e F_{\text {tip }} r_{\text {tip }}\right)$ seems to underestimate $U_{\infty}$ at a point far from the emitter, when the emitter is needle-shaped with a shank or it is a pyramid-shaped emitter with an integrated electron extraction gate. ${ }^{67}$ Nevertheless, we can conclude that the transverse acceleration of the field emission electron beam leads to the increase in the transverse spread of the beam by a factor that is less than 2. This leads to the proportional increase in the average transverse velocity and the intrinsic emittance of the beam compared to the planar cathode case. This is not against the conservation of the intrinsic transverse emittance (and the average transverse energy) of a particle beam, since the transverse emittance strictly conserves only when the transverse motion is separated from the longitudinal motion ${ }^{44,68}$ that is not the case of electron motion under acceleration in the spherical potential.

The ratio of the transverse spread to the propagation distance of the wave function calculated for the model planar cathode (57) was equal to (55) when $a_{s}=0$, if we equate $\left(F_{t i p} r_{t i p}\right)$ to $U_{0}$. However, (57) also indicates the significant reduction of the transverse spread by the factor $\sigma_{0} / a_{s}$ (especially when $a_{s} \gg \sigma_{0}$ ). This opposing effect against the deflection by the transverse electric field is not anticipated from the electron optic analysis and related to the coherent wave propagation of the field emission electrons when those are fully coherent. This conclusion suggests that the intrinsic transverse emittance of field emitters is smaller than the value predicted by classical analysis. One may be able to observe this effect, for example, when the electron coherent length in the solid is enhanced at low temperature. ${ }^{17}$ For a single-tip needle-shaped field emitter, the predicted reduction of the transverse spread may have only a marginal effect, since its intrinsic emittance is already extremely small even under the laser driven emission mode. ${ }^{69}$ However, such a coherence-induced reduction of the transverse spread of field emission beam will have a significant influence on double-gate FEAs for accelerator applications. Because of the finite array pitch and the finite array side due to the large emission current requirement, the reduction of the transverse beam spread is the key to increase the beam brightness of double-gate FEAs. This is especially the case since the average transverse energy of $0.1-0.2 \mathrm{eV}$ of the field emission beam as determined by the Fermi-Dirac distribution in typical metals is of the same magnitude as the minimal average transverse energy (= intrinsic transverse energy per unit area) of the state-of-the-art photocathode at high current operation limited by the electron temperature. ${ }^{70,71}$ We point out that the average transverse energy equal to $0.12 \pm 0.06 \mathrm{eV}$ evaluated from the experimentally obtained intrinsic transverse emittance of a double-gate FEA under maximally collimated beam condition at room temperature was in agreement with the theoretical value of $0.19 \pm 0.01 \mathrm{eV}$ calculated from (41) with the tip field of $4.4 \pm 0.1 \mathrm{GV} / \mathrm{m}$ estimated from the fitting of $I-V,{ }^{45}$ and this is in line with the possible reduction of the intrinsic emittance by the finite coherence of the emitter tip. Nevertheless, the propagation of field emission electron wave in non-planar potential has to be investigated further and carefully compared with experiments in the future research.

\section{F. Further discussions}

For more quantitative analysis of the wave front of the field emission beam emitted from actual emitters, several important physics, that are partly common to the theory of the field emission current-voltage characteristics as summarized recently, ${ }^{72}$ will have to be taken into account. It is also important to note that the atomic scale corrugation and the lattice structure of the material are important at the field emitter surface. ${ }^{23,73-77}$ In addition, even for emitters with $r_{t i p}$ of tens of nanometer-scale, the quantum confinement effect on the electronic states at the emitter tip may affect the emission process for semiconductor emitters. ${ }^{78}$ The uniform emitter surface may be a valid assumption in some cases, but emitters with the tip apex radius of curvature $r_{\text {tip }}$ of a few nanometers down to single-atom ${ }^{75}$ will require the consideration of the bulk and surface band structure. Comparing to the impact of such three-dimensional-shape effects on the current-voltage characteristics of field emitters, ${ }^{79-82}$ their influences on the wave function are not much studied yet. Interestingly, the estimated full width at the half maximum of the wave function at the surface of a "point" emitter equal to $0.7 \mathrm{~nm}$ (at the surface field of $5 \mathrm{GV} / \mathrm{m}$, with the corresponding $\sigma_{0}=0.42 \mathrm{~nm}$ ) is consistent with the sub-nanometer resolution of the field emission microscope for sub-nanometer- $r_{t i p}$ emitters based on the electron optical analysis ${ }^{23,83}$ and agrees with the values observed in the experiments. ${ }^{7,84-86}$ Nevertheless, for comprehensive elucidation of the wave function of the field emission electrons from atomic-scale emitters, further research that takes into account the influence of the atomic orbitals ${ }^{87}$ and the energy spectra $^{76,85,88,89}$ will be required.

\section{SUMMARY AND CONCLUSION}

In summary, motivated by the recent experimental finding of a small intrinsic average transverse energy of field emission beam, ${ }^{45}$ we investigated its implication on the transverse structure of the wave function of field emission electron beam for a planar cathode as a model. We found that the average transverse energy determines the minimal transverse spatial spread of the field emission electron beam at the cathode surface. We calculated the propagation of the electron wave function from the field emitter tip apex by applying the WKB approximation and showed that the wave function converges to a form similar to the optical Gaussian beam. Consequently, when an electron is emitted from a finite but fully coherent emission area, the transverse spatial spread of the field emission beam is significantly narrower than that of the beam emitted from a point source. The comparison of the transverse spread of the wave function with the deflection angle (blurring fall-off half-angle) of the electron orbit from the axial direction due to the finite transverse energy in the spherical emitter case suggests that the quantum mechanical interference may partially cancel the transverse deflection and reduce the intrinsic transverse emittance of field emitters from the value determined from the average transverse energy and the additional transverse deflection by the spherical cathode potential near the tip apex. An extension of the present theory on a curved surface as well as the 
inclusion of the lattice effect and the finite coherence over the field emitter tip surface is highly desired and is the subject of future research.

\section{ACKNOWLEDGMENTS}

The author acknowledges A. Patterson for the discussion on the three-dimensional electronic states of field emitters, and to T. A. Jung for the discussion on the electron tunneling from nanoscale metal tips. A special thanks to T. Latychvskaia for enlightening discussions on the electron coherence and their applications, and the critical reading of the manuscript, and to C. Metzner for the discussion on the conservation of the transverse momentum.

${ }^{1}$ A. V. Crewe, Science 154, 729-738 (1966).

${ }^{2}$ J. C. H. Spence, High-Resolution Electron Microscopy, 4th ed. (Oxford University Press, Oxford, UK, 2017).

${ }^{3}$ Y. Fujiyoshi, Adv. Biophys. 35, 25 (1998).

${ }^{4}$ A. Tonomura, N. Osakabe, T. Matsuda, T. Kawasaki, J. Endo, S. Yano, and H. Yamada, Phys. Rev. Lett. 56, 792 (1986).

${ }^{5}$ H.-W. Fink, W. Stocker, and H. Schmid, Phys. Rev. Lett. 65, 1204 (1990).

${ }^{6}$ A. Eisele, B. Voelkel, M. Grunze, and A. Golzhauser, Z. Phys. Chem. 222, 779-787 (2008).

${ }^{7}$ M. Yamashita, M. R. Leyden, H. Adaniya, M. P. Cheung, T. Hirai, Y. Qi, and T. Shintake, Microscopy 66, 261 (2017).

${ }^{8}$ P. A. Midgley and R. E. Dunin-Borkowski, Nat. Mater. 8, 271 (2009).

${ }^{9}$ J. Verbeeck, H. Tian, and P. Schattschneider, Nature 467, 301 (2010).

${ }^{10}$ B. J. McMorran, A. Agrawal, I. M. Anderson, A. A. Herzing, H. J. Lezec, J. J. McClelland, and J. Unguris, Science 331, 192 (2011).

${ }^{11}$ R. Shiloh and A. Arie, Ultramicroscopy 177, 30 (2017).

${ }^{12}$ M. Born and E. Wolf, Principles of Optics, 6th ed. (Pergamon Press Ltd., Oxford, 1980), p. 508.

${ }^{13}$ E. Ruska, Z. Phys. 83, 684 (1933).

${ }^{14}$ T. E. Everhard, J. Appl. Phys. 38, 4944 (1967).

${ }^{15}$ W. Qian, M. R. Scheinfein, and J. C. H. Spence, J. Appl. Phys. 73, 7041-7045 (1993).

${ }^{16}$ A. H. V. van Veen, C. W. Hagen, J. E. Barth, and P. Kruit, J. Vac. Sci. Technol. B 19, 2038 (2001).

${ }^{17}$ B. Cho, T. Ichimura, R. Shimizu, and C. Oshima, Phys. Rev. Lett. 92, 246103 (2004).

${ }^{18}$ D. Ehberger, J. Hammer, M. Eisele, M. Krüger, J. Noe, A. Högele, and P. Hommelhoff, Phys. Rev. Lett. 114, 227601 (2015).

${ }^{19}$ G. Schütz, A. Rembold, A. Pooch, S. Meier, P. Schneeweiss, A. Rauschenbeutel, A. Günther, W. T. Chang, I. S. Hwang, and A. Stibor, Ultramicroscopy 141, 9 (2014).

${ }^{20}$ L. W. Swanson and G. A. Schwind, "A review of the cold field electron cathode," in Advances in Imaging and Electron Physics (Elsevier 2009), Vol. 159, p. 80.

${ }^{21}$ R. G. Forbes, "The theory of bright field electron and field ion emission sources," in Nanofabrication Using Focused Ion and Electron Beams: Principles and Applications, edited by I. Utke, S. Moshkalev, and P. Russell (Oxford University Press, Oxford, 2012).

${ }^{22}$ P. W. Hawkes and E. Kasper, "Principles of electron optics, Vol. II," Applied Geometrical Optics, 2nd ed. (Academic Press, London, 2018), Chap. 45.

${ }^{23}$ R. H. Good, Jr. and E. W. Müller, in Field Emission, Handbuch Der Physik Vol. XXI, edited by S. Fluegge (Springer-Verlag, Berlin, 1956), pp. 176-231.

${ }^{24}$ M. Krüger, M. Schenk, and P. Hommelhoff, Nature 475, 78 (2011).

${ }^{25}$ G. Henrik, D. R. Solli, M. Gulde, and C. Ropers, Nature 483, 190 (2012).

${ }^{26}$ G. Wachter, C. Lemell, J. Burgdörfer, M. Schenk, M. Krüger, and P. Hommelhoff, Phys. Rev. B 86, 035402 (2012).

${ }^{27}$ J. Schötz, S. Mitra, H. Fuest, M. Neuhaus, W. A. Okell, M. Förster, T. Paschen, M. F. Ciappina, H. Yanagisawa, P. Wnuk, P. Hommelhoff, and M. F. Kling, Phys. Rev. A 97, 013413 (2018).

${ }^{28}$ G. Pozzi, Optik 77, 69 (1987).

${ }^{29}$ L. Mandel and E. Wolf, Optical Coherence and Quantum Optics (Cambridge University Press, 1995), p. 272.

${ }^{30} \mathrm{P}$. W. Hawkes and E. Kasper, "Principles of electron optics, Vol. III," in Wave Optics, 2nd ed. (Academic Press, London, 1994), Chap. 66.
${ }^{31}$ B. D. Patterson, R. Abela, H.-H. Braun, U. Flechsig, R. Ganter, Y. Kim, E. Kirk, A. Oppelt, M. Pedrozzi, S. Reiche, L. Rivkin, T. Schmidt, B. Schmitt, V. N. Strocov, S. Tsujino, and A. F. Wrulich, New J. Phys. 12, 035012 (2010).

${ }^{32}$ P. Emma, R. Akre, J. Arthur, R. Bionta, C. Bostedt, J. Bozek, A. Brachmann, P. Bucksbaum, R. Coffee, F.-J. Decker, Y. Ding, D. Dowell, S. Edstrom, A. Fisher, J. Frisch, S. Gilevich, J. Hastings, G. Hays, P. Hering, Z. Huang, R. Iverson, H. Loos, M. Messerschmidt, A. Miahnahri, S. Moeller, H.-D. Nuhn, G. Pile, D. Ratner, J. Rzepiela, D. Schultz, T. Smith, P. Stefan, H. Tompkins, J. Turner, J. Welch, W. White, J. Wu, G. Yocky, and J. Galayda, Nat. Photonics 4, 641 (2010).

${ }^{33}$ T. Ishikawa, H. Aoyagi, T. Asaka, Y. Asano, N. Azumi, T. Bizen, H. Ego, K. Fukami, T. Fukui, Y. Furukawa, S. Goto, H. Hanaki, T. Hara, T. Hasegawa, T. Hatsui, A. Higashiya, T. Hirono, N. Hosoda, M. Ishii, T. Inagaki, Y. Inubushi, T. Itoga, Y. Joti, M. Kago, T. Kameshima, H. Kimura, Y. Kirihara, A. Kiyomichi, T. Kobayashi, C. Kondo, T. Kudo, H. Maesaka, X. M. Maréchal, T. Masuda, S. Matsubara, T. Matsumoto, T. Matsushita, S. Matsui, M. Nagasono, N. Nariyama, H. Ohashi, T. Ohata, T. Ohshima, S. Ono, Y. Otake, C. S, T. Sakurai, T. Sato, K. Sawada, T. Seike, K. Shirasawa, T. Sugimoto, S. Suzuki, S. Takahashi, H. Takebe, K. Takeshita, K. Tamasaku, H. Tanaka, R. Tanaka, T. Tanaka, T. Togashi, K. Togawa, A. Tokuhisa, H. Tomizawa, K. Tono, S. Wu, M. Yabashi, M. Yamaga, A. Yamashita, K. Yanagida, C. Zhang, T. Shintake, H. Kitamura, and N. Kumagai, Nat. Photonics 6, 540 (2012).

${ }^{34}$ R. J. D. Miller, Ann. Rev. Chem. 65, 583-604 (2014).

${ }^{35}$ C. Lee, P. D. Kanungo, V. Guzenko, P. Helfenstein, R. J. D. Miller, and S. Tsujino, J. Vac. Sci. Technol. B 33, 03C111 (2015).

${ }^{36}$ C. Lee, S. Tsujino, and R. J. D. Miller, Appl. Phys. Lett. 113, 013505 (2018).

${ }^{37}$ P. Hommelhoff, Y. Sortais, A. Aghajani-Talesh, and M. A. Kasevich, Phys. Rev. Left. 96, 077401 (2006).

${ }^{38}$ C. Ropers, D. R. Solli, C. P. Schulz, C. Lienau, and T. Elsaesser, Phys. Rev. Lett. 98, 043907 (2007).

${ }^{39}$ H. Yanagisawa, C. Hafner, P. Doná, M. Klöckner, D. Leuenberger, T. Greber, J. Osterwalder, and M. Hengsberger, Phys. Rev. B 81, 115429 (2010).

${ }^{40}$ A. Mustonen, P. Beaud, E. Kirk, T. Feurer, and S. Tsujino, Sci. Rep. 2, 915 (2012).

${ }^{41}$ P. Helfenstein, A. Mustonen, T. Feurer, and S. Tsujino, Appl. Phys. Express. 6, 114301 (2013).

${ }^{42}$ Y. Oh, A. Mustonen, T. Feurer, and S. Tsujino, J. Vac. Sci. Technol. B 33, $03 \mathrm{C} 112$ (2015).

${ }^{43}$ S. Lee, J. Choi, H. Song, T. Y. Kang, J. Oh, and K. Kim, J. Lightwave Technol. 34, 4023 (2016).

${ }^{44} \mathrm{M}$. Reiser, Theory and Design of Charged Particle Beams (Wiley-VCH Verlag, 2014)

${ }^{45}$ S. Tsujino, P. Das Kanungo, M. Monshipouri, C. Lee, and R. J. D. Miller, Nat. Commun. 7, 13976 (2016).

${ }^{46}$ C. J. Edgcombe, Ultramicroscopy 110, 1454 (2010).

${ }^{47}$ C. J. Edgcombe, Adv. Imaging Electron Phys. 162, 77 (2010).

${ }^{48}$ R. G. Forbes and J. H. B. Deane, Proc. R. Soc. A 463, 2907-2927 (2007).

${ }^{49}$ E. L. Murphy and R. H. Good, Phys. Rev. 102, 1464-1473 (1956).

${ }^{50}$ K. L. Jensen, J. Vac. Sci. Technol. B 21, 1528 (2003).

${ }^{51}$ D. H. Dowell and J. F. Schmerge, "Quantum efficiency and thermal emittance of metal photocathodes," Phys. Rev. Spec. Top.-Accel. Beams 12, 074201 (2009).

${ }^{52}$ P. W. Hawkes and E. Kasper, "Principles of electron optics, Vol. II," in Applied Geometrical Optics, 2nd ed. (Academic Press, London, 2018), Chap. 44.

${ }^{53}$ L. W. Sanson and A. E. Bell, "Recent advances in field electron microscopy of metals," in Advances in Electronics and Electron Physics, edited by L. Marton (Academic Press, New York, 1973), Vol. 32, p. 200, 300.

${ }^{54}$ L. I. Schiff, Quantum Mechanics, 3rd. ed. (McGrow-Hill Book Co, Singapore, 1968), p. 270.

${ }^{55}$ L. D. Laudau and E. M. Lifshitz, Quantum Mechanics (Pergamon Press, Ltd., 1977), p. 74.

${ }^{56} \mathrm{~S}$. Moriguchi, K. Utagawa, and S. Hitomatsu, Iwanami Mathematical Formula III (Iwanami, Tokyo, 1960), p. 200.

${ }^{57}$ F. W. J. Olver, A. B. Olde Daalhuis, D. W. Lozier, B. I. Schneider, R. F. Boisvert, C. W. Clark, B. R. Miller, and B. V. Saunders, see http:// dlmf.nist.gov/ for Formula 10.22.51 in NIST Digital Library of Mathmatical Functions; Release 1.0.17 of 2017-12-22.

${ }^{58} \mathrm{~T}$. Latychevskaia, Ultramicroscopy 175, 121 (2017).

${ }^{59}$ R. Gomer, Field Emission and Field Ionization (American Institute of Physics, New York, 1993), p. 32.

${ }^{60}$ J. J. Sáenz and R. García, Appl. Phys. Lett. 65, 3022 (1994). 
${ }^{61}$ T. L. Kirk, U. Ramsperger, and D. Pescia, J. Vac. Sci. Technol. B 27, 152 (2009).

${ }^{62}$ D. A. Zanin, L. G. De Pietro, Q. Peter, A. Kostanyan, H. Cabrera, A. Vindigni, T. Bähler, D. Pescia, and U. Ramsperger, Proc. R. Soc. A 472, 20160475 (2016).

${ }^{63}$ D. A. Zanin, H. Cabrera, L. G. De Pietro, M. Pikulski, M. Goldmann, U. Ramsperger, D. Pescia, and J. P. Xanthakis, "Fundamental aspects of nearfield emission scanning electron microscopy," in Advances in Imaging and Electron Physics (Elsevier, 2012), Vol. 170, p. 227.

${ }^{64}$ A. Yariv, Quantum Electronics, 3rd ed. (John Wiley \& Sons, Inc., 1989), p. 116.

${ }^{65}$ J. W. Goodman, Introduction to Fouier Optics, 3rd ed. (Roberts \& Co., Englewood, Colorado, 2005), Chap. 4.

${ }^{66} \mathrm{P}$. W. Hawks and E. Kasper, "Principle of electron optics," in Wave Optics (Academic Press, London, 1994), Vol. 3, Chap. 59.

${ }^{67}$ The energy ( $\left.e F_{\text {tip }} r_{\text {tip }}\right)$ is equal to the kinetic energy of an electron $U_{\infty}$ at infinity in the case of the spherical emitter and the concentric spherical acceleration potential. In general, $\left(e F_{\text {tip }} r_{\text {tip }}\right)$ underestimates $U_{\infty}$ at a point far from the emitter, e.g., when the emitter is a needle-shaped with a shank or a pyramid-shape emitter with an integrated electron extraction gate. We found from particle tracking simulations in electrostatic potentials calculated by finite-element simulation that the ratio $U_{\infty} /\left(e F_{\text {tip }} r_{\text {tip }}\right)$ is in the range of 2-6.

${ }^{68} \mathrm{P}$. W. Hawkes and E. Kasper, "Principles of electron optics, Vol. II," in Applied Geometrical Optics, 2nd ed. (Academic Press, London, 2018), Chap. 48.

${ }^{69}$ A. Feist, N. Bach, N. R. da Silva, T. Danz, M. Möller, K. E. Priebe, T. Domröse, J. G. Gatzmann, S. Rost, J. Schauss, S. Strauch, R. Bormann, M. Sivis, S. Schäfer, and C. Ropers, "Ultrafast transmission electron microscopy using a laser-driven field emitter: Femtosecond resolution with a high coherence electron beam," Ultramicroscopy 176, 63 (2017).
${ }^{70}$ J. Feng, J. Nasiatka, W. Wan, S. Karkare, J. Smedley, and H. A. Padmore, "Thermal limit to the intrinsic emittance from metal photocathodes," Appl. Phys. Lett. 107, 134101 (2015).

${ }^{71}$ J. Maxon, P. Musumeci, L. Cultrera, S. Karkare, and H. Padmore, "Ultrafast laser pulse heating of metallic photocathodes and its contribution to intrinsic emittance," Nucl. Instrum. Methods Phys. A 865, 99 (2017).

${ }^{72}$ R. G. Forbes, Nanotechnology 23, 095706 (2012).

${ }^{73}$ Y. Saito, K. Hata, and T. Murata, Jpn. J. Appl. Phys., Part 2 39, L271 (2000).

${ }^{74}$ C. Oshima, K. Mastuda, T. Kona, Y. Mogami, M. Komaki, Y. Murata, T. Yamashita, T. Kuzumaki, and Y. Horiike, Phys. Rev. Lett. 88, 038301 (2002).

${ }^{75}$ H.-W. Fink, IBM J. Res. Develop. 30, 460 (1986).

${ }^{76}$ V. T. Binh, S. T. Purcell, N. Garcia, and J. Doglioni, Phys. Rev. Lett. 69, 2527 (1992).

${ }^{77}$ K. Murakami, F. Wakaya, and M. Takai, J. Vac. Sci. Technol. 25, 1310 (2007).

${ }^{78}$ A. A. Patterson and A. I. Akinwande, J. Appl. Phys. 114, 234303 (2013).

${ }^{79}$ J. He, P. H. Cutler, N. M. Miskovsky, T. E. Feuchtwang, T. E. Sullivan, and M. Chung, Surf. Sci. 246, 348 (1991).

${ }^{80}$ R. C. Smith and S. R. P. Silva, Appl. Phys. Lett. 94, 133104 (2009).

${ }^{81}$ A. Kyritsakis and J. P. Xanthakis, Proc. R. Soc. A 471, 20140811 (2015).

${ }^{82}$ D. Biswas and R. Ramachandran, Phys. Plasmas 24, 073107 (2017).

${ }^{83}$ D. J. Rose, J. Appl. Phys. 27, 215 (1956).

${ }^{84}$ E. Rokuta, H.-S. Kuo, T. Itagaki, K. Nomura, T. Ishikawa, B.-L. Cho, I.-S. Hwang, T. T. Tsong, and C. Oshima, Surf. Sci. 602, 2508 (2008).

${ }^{85}$ M. Rezeq, C. Joachim, and N. Chandrasekhara, Microelectron. Eng. 86, 996 (2009).

${ }^{86}$ M. Rezeq, Microelectron. Eng. 102, 2 (2013).

${ }^{87}$ H. Choi, R. C. Longo, M. Huang, J. N. Randall, R. M. Wallace, and K. Cho, Nanotechnology 24, 105201 (2013).

${ }^{88}$ N. D. Lang, A. Yacoby, and Y. Imry, Phys. Rev. Lett. 63, 1499 (1989).

${ }^{89}$ N. Ernst, J. Unger, H.-W. Fink, M. Grunze, H. U. Müller, B. Völkl, M. Hofmann, and C. Wöll, Phys. Rev. Lett. 70, 2503 (1993). 